\title{
Monolayer Structure of Supramolecular Antagonistic Salt Aggregates
}

\author{
David Jung, ${ }^{\dagger, \ddagger}$ Jens Harting, ${ }^{*, \dagger, \uparrow}$ and Marcello Sega*, \\ $\dagger$ †elmholtz Institute Erlangen-Nürnberg for Renewable Energy, Forschungszentrum Jülich, \\ Fürther Straße 248, 90429 Nürnberg, Germany. \\ $\ddagger$ Department of Physics, Friedrich-Alexander-Universität Erlangen-Nürnberg, Fürther \\ Straße 248, 90429 Nürnberg, Germany. \\ \Department of Chemical and Biological Engineering and Department of Physics, \\ Friedrich-Alexander-Universität Erlangen-Nürnberg, Fürther Straße 248, 90429 Nürnberg, \\ Germany. \\ E-mail: j.harting@fz-juelich.de; m.sega@fz-juelich.de \\ Phone: +49 911 32169-113; +49 911 32169-102
}

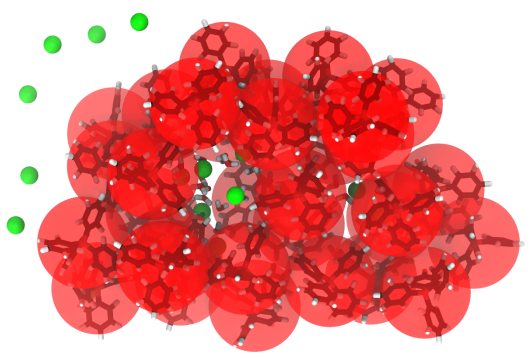

Figure S1: Close-up of a vesicle-like structure formed from a leaflet wrapped around several enclosed $\mathrm{Na}^{+}$due to thermal fluctuations at $10 \% 3-\mathrm{MP}$ and $140 \mathrm{mM}$ salt. $\mathrm{BPh}_{4}^{-}$is highlighted by transparent $0.5 \mathrm{~nm}$ radius spheres, $\mathrm{Na}^{+}$is shown as green spheres, water solvating vesicle-enclosed water is rendered as sticks. Remaining empty space in vesicle is filled with 3-MP. 


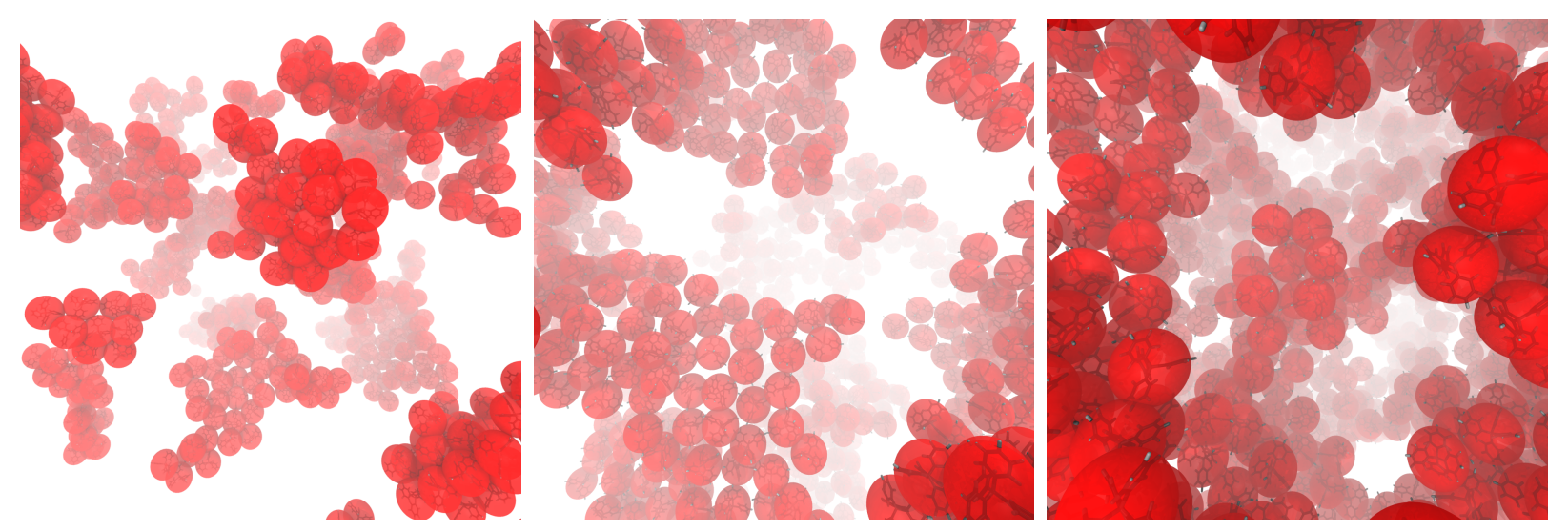

Figure S2: Details of snapshots from equilibrated atomistic simulations of ternary mixtures (large system size, edge length $\approx 20 \mathrm{~nm}$ ) at salt concentrations of 140 (left panel), 210 (middle panel) and $500 \mathrm{mM}$ (right panel). For clarity, only $\mathrm{BPh}_{4}^{-}$ions are shown. The volume fraction of 3-MP was $10 \%$ (left), $15 \%$ (middle), and $15 \%$ (right).

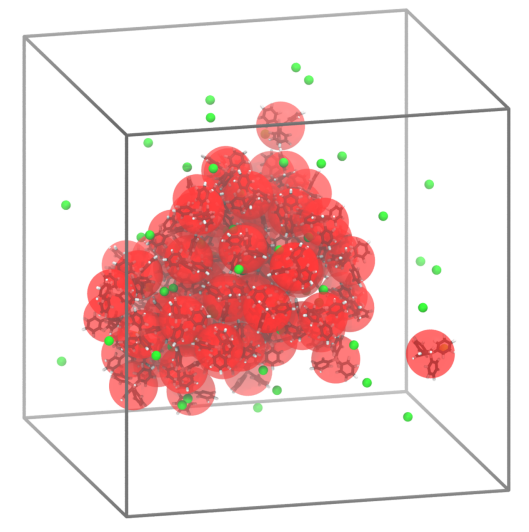

Figure S3: Atomistic simulation at 15\% 3-MP and $250 \mathrm{mM}$ salt with $\mathrm{Na}^{+}$Lennard-Jones parameter $\epsilon$ decreased 20 -fold. Due to a reduced hydration shell size, $\mathrm{Na}^{+}$can infiltrate $\mathrm{BPh}_{4}^{-}$ aggregates. Cations not shown to scale. 

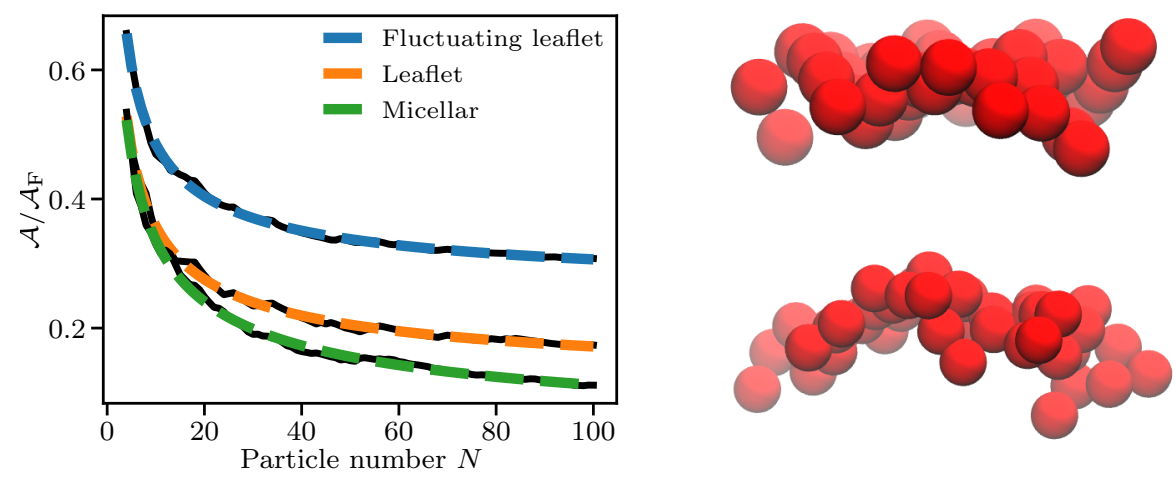

Figure S4: Left: Power law fits (dashed) to numerically calculated ASA per particle (full lines) in different aggregate types of size $N$. Right: Comparison of generated fluctuating leaflet morphology (top) to simulated leaflet (bottom) from the left pane of Fig. S2. 